\title{
DGP Newsletter September 2009
}

Redaktion: U. Costabel, Essen

Liebe Kolleginnen und Kollegen, im September werden in vielen Orten Deutschlands die letzten Vorbereitungen zum Deutschen Lungentag getroffen, der traditionell am Monatsende stattfindet. Sie finden hier die Ankündigung des 12. Deutschen Lungentages und einen Bericht von Gerda Cromberg über die Beteiligung des Berufskollegs der Stadt Herne als Schulveranstalter seit 1998 am Deutschen Lungentag mit dem Schwerpunktthema der Tabakprävention.

Ulrich Costabel

\section{Deutscher Lungentag 2009 \\ $\nabla$}

Die bundesweite Jahresveranstaltung des Deutschen Lungentages e.V. ist im Monat September inzwischen zu einer festen Größe geworden.

Der 12. Deutsche Lungentag steht im Jahr 2009 unter dem Motto „Wellness für die Lunge“. Er findet am 26. September 2009 statt.

An zahlreichen Orten der Bundesrepublik Deutschland bieten Facharztpraxen, Kliniken, Krankenhäuser, Krankenkassen, Ämter, Apotheken und Behörden Aktionen, Patientenforen, Fachvorträge, Informationsstände für die Bevölkerung an. Als Beispiel werden dabei Themen wie Luftbelastung (Rauchen, Passivrauchen, Stäube), medikamentöse und nicht-medikamentöse Hilfen sowie Lungensport besprochen.

Nähere Informationen zu den einzelnen Veranstaltungsorten und den vielfältigen Angeboten der zahlreichen Anbieter u.a.m. können auf den Webseiten des Deutschen Lungentages e.V. über www.lungentag.de ab September abgefragt werden.

Prof. K. Ch. Bergmann Vorsitzender Deutscher Lungentag e.V.

\section{Organisation eines Lungentages am Beispiel des Berufskollegs für Wirtschaft und Verwaltung der Stadt Herne $\nabla$}

Das Berufskolleg ist seit 1998 Schul-Veranstalter des 1-mal jährlich bundesweit wiederkehrenden Deutschen Lungentages (s. OPUS/Klinik/Praxis/ ADIZ).

Schul- und Projektleitung:

Oberstudiendirektor, Heribert Gathmann Koordinator f. OPUS NRW - Stadt Herne:

Peter Scholz

Anleitung Projekt „Umwelt-Lunge-Sucht: Modell Klasse 2000 - Tabakprävention mit PowerPoint und Internet/Lungensport“ Gerda Cromberg, FL, Ansprechpartnerin und Mitglied der außerschulischen Partner:

- Deutschen Atemwegsliga e.V.

- Arbeitsgemeinschaft Lungensport in Deutschland e.V.

- Deutsche Gesellschaft für Nikotin- und Tabakforschung e.V.

- Ärztlicher Arbeitskreis „Rauchen“ e.V.

\section{Projektbeginn}

Auslöser waren Klassenkonferenzen wegen hoher Fehlzeiten von Schülern mit Allergien, Asthma, Mukoviszidose (Raucher). Durch den damaligen 1. Vorsitzenden der Deutschen Atemwegsliga, Prof. Dr. Linus Geisler ${ }^{1}$, kannte ich die schulische Prävention der Liga, deren soziales Engagement für plötzlich in Not geratene Mukoviszidosekranke, wurde 1994 Mitglied, erhielt Informationsmaterial $^{2}$ für meinen Unterricht nach „Klasse 2000“ und organisierte Arztvorträge („Tag-der-offenenTür“").

\footnotetext{
${ }^{1}$ „Arzt und Patient - Begegnung im Gespräch - Wirklichkeit und Wege“, pmi Verlag AG und „Leben mit Asthma und Lungenemphysem“, Joop-Verlag - Autor Prof. Dr. Linus Geisler

2 Positionspapiere der Atemwegsliga; 2000 „Pneumologie“ Empfehlungen der Dt. Atemwegsliga zum Sport und körperlichen Training bei Patienten mit obstruktiven Atemwegskrankheiten
} 


\section{Projektverlauf}

1998 Erster Lungentag mit Lungenfachärztin ${ }^{3}$

1999 Regionales Kooperationsprojekt der

Dt. Atemwegsliga mit der Stadt Herne und Mitgliedschaft OPUS Netzwerk NRW. Meine Öffentlichkeitsarbeit: Besuch von Ärztefortbildungen ${ }^{4,5,6}$; Veröffentlichungen und Berichte zur Projektentwicklung ${ }^{7}$ - www.herne.de; google.de - Gerda Cromberg

- 2000 Erste Veröffentlichung der Auszüge aus PowerPointPräsentationen meiner Klassen durch den Deutschen Lungentag

-2001/02 Erstmals Vorstellung des Lungentag-Projektes mit Lungensport ${ }^{8}$ für alle, durch Lungenfacharzt ${ }^{9}$ und Mothopäden der Reha-Klinik-Hagen-Ambrock (OPUS-Lehrerfortbildung-Herne $)^{10}$

- 2003 Vorstellung meines projektbezogenen Unterrichts ${ }^{11}$ _ Tabakprävention im EDV-Unterricht ${ }^{12}$ nach Modell: Klasse 2000 - Nichtraucherschutzgesetz/Arbeitsstättenverordnung $\S 3$ a. (OPUS-Lehrerfortbildungen-Herne/Haltern) ${ }^{13}$

- 2005 Postervorstellungen: Nikotinkonferenz Erfurt ${ }^{14,15}$ : Lehrerfortbildung Schwerin (Rauchfreie Schule) ${ }^{16}$; WHOKonferenzen Kollaborationszentrum für Tabakkontrolle, Heidelberg. Workshop/Berlin ${ }^{18,17}$

2006 Kombination: Lungentag/Gesundheitstag/Herne ${ }^{19}$

\section{Zertifizierung}

2006/07 Zu Beginn meines Weiterbildungs-Fernstudiums „Gesundheitsförderung in Städten und Gemeinden“, an der FH Magdeburg, bewarb ich mich mit der Schulleitung und dem Projekt um den Deutschen Präventionspreis 2007. Laufzeit: 2007-2011. März 2007 erhielten wir die Einstiegsurkunde für den Wettbewerb.

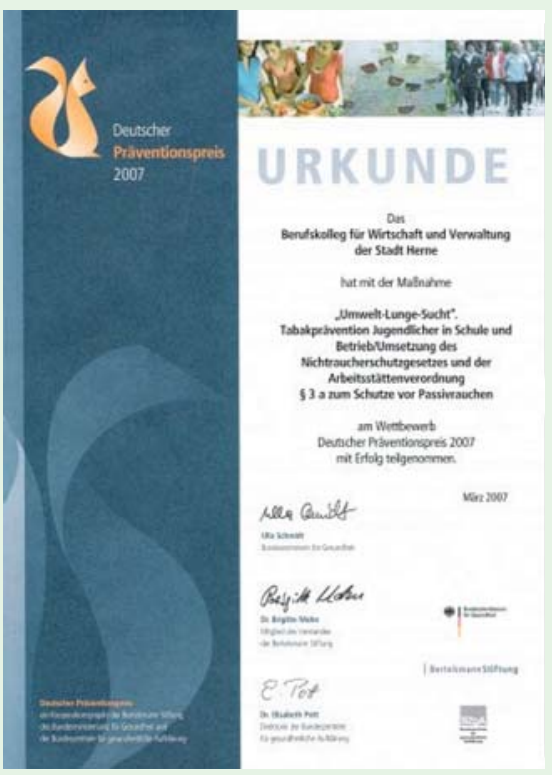

2007 Thema meiner Hausarbeit: „Organisation eines Lungentages mit außerschulischen Partnern“, siehe Eintrag in die Datenbank Berlin „Gesundheitliche Chancengleichheit“ Gesundheit Schule - Beruf". Veröffentlichung des Projektes mit dem Modell: „Klasse 2000“/Tabakprävention mit PowerPoint/Internet/Lungensport für alle: Datenbank des OPUS-Netzwerks für Bildung und Gesundheit NRW.

3 Frau Dr. Redecker, Ruhrlandklinik Essen

${ }^{4}$ Mein Besuch des „Train-the-trainer-Seminars zur Schulung von Patienten, Akademie der Ärztekammer Westf.-Lippe/Nov., Leitung Prof. Dr. Rühle, Reha-Klinik Hagen-Ambrock; 2000, weitere Veröffentlichungen über Deutsche Nikotin-Konferenzen Erfurt; Lungensport-Lehrerfortbildung in der Klinik Hamburg-Großhansdorf

5 Journal der Atemwegsliga; der AG Lungensport in Deutschland „Kinderluftpost"

${ }^{6}$ Vorsitzender der Deutschen Atemwegsliga und der AG Lungensport in Deutschland, Prof. Dr. med. Heinrich Worth/Asthma-Leitlinie//COPD-Leitlinie - Koordinatorin: Frau Dr. med. Uta Butt

7 Bericht im vLw - 2006-01-11 - Die Kaufmännische Schule - „Gesundheitsförderung und Raucherprävention - 7 Jahre Erfahrung am Berufskolleg f. Wirtschaft u. Verwaltung der Stadt Herne“ - www.google.de - Gerda Cromberg

8 Vorsitzender der Dt. Atemwegsliga und der AG Lungensport in Deutschland, Prof. Dr. med. Worth, u. a. „Mit Asthma komme ich klar“

92002 Vorstellung des Atemwegsliga-Lungentagprojektes von Dr. Wiemann und mir bei der Universität Bochum, Institut für Physiologie, Frau Prof. Dr. Schläfke

10 Veröffentlichung meines Projektes in der Homepage des BK

11 Beispiel zur curricularen Einordnung in meinem Unterricht -Hausarbeit zum Päd. Lehrgang des RP Köln, Juni 2003: „Projektarbeit zum Thema Gesundheitsförderung mit dem besonderen Aspekt der Medienförderung“

12 „Konstruktivismus, die neue Perspektive im (Sach-) Unterricht“ Autoren: Prof. Dr. Klaus Klein, Ulrich Oettinger, Universität Köln

13 Universität Bochum, Institut für Physiologie, Frau Prof. Dr. Schläfke - Präsentation Nichtraucherschutzgesetz unter www.atemwegsliga.de (Aktuelles)

14 „Zusammenfassung der Vorträge zur 8. Deutschen Nikotinkonferenz in Erfurt - „Passivrauchen Rauchen in der Schule - Möglichkeiten der Raucherentwöhnung“ Autor: Prof. Dr. med. Knut-Olaf Haustein. Mein Bericht dazu: „Gesundheitsförderung - Raucherprävention in Schulen“ - Curriculare Einordnung in den Unterricht: Lernfeld: Betriebliche Informationssysteme (EDV) - Arbeits- und Gesundheitsschutz - www.safety1st und www. sozialpolitik.com - Literatur: u. a. Prof. Dr. Batra, „Nichtraucher in 6 Wochen“, Herausgeber: Arbeitskreis Raucherentwöhnung, Universität f. Psychiatrie und Psychotherapie Tübingen, Bundeszentrale f. gesundheitliche Aufklärung.

15 Veröffentlichte Berichte in Lungentag „SPEZIAL 2005“ und Suchtmed 7 (1) 2005 Risikofaktor Rauchen - Gesundheitsaufklärung in der Schule - Lungentag 2006

16 Moderatorin auf der vom Landesinstitut für Schule und Ausbildung Mecklenburg-Vorpommern - anerkannten Fachtagung in Schwerin - Rauchen - Gesellschaftliche oder private Verantwortung?

17 Teilnahme am Workshop des Kooperationsprojektes des Bundesministeriums für Bildung und Forschung mit dem Wissenschaftszentrum für Sozialforschung und Gesundheit, Berlin.

18 Betreuung des Lungentages von 2003 bis 2007: Frau Dr. Khanavkar, Augusta-Krankenanstalten, Bochum. 2007 erstmals mit Beteiligung der Patientenliga Atemwegserkrankungen, Hagen.

19 Mein Bericht über den Lungensport für alle am BK Herne. Empfänger: Heinrich-Heine-Universität Düsseldorf 


\section{Lungentag 2007 (Muster) $^{20}$}

1. Meine Klassen erstellen im projektbezogenen EDV-Unterricht - Modell: Klasse 2000 - einzelne PowerPoint-Präsentationen zur Tabakprävention und erhalten seit 2007 dafür eine Bescheinigung des Deutschen Lungentages. www.lungentag.de

2. Die vom Dt. Lungentag und dem Vorsitzenden, Prof. Dr. Bergmann, veröffentlichte Demo-CD, dient als Einstieg in den Aktionstag am $\mathrm{BK}^{21}$.

3. Neben der Informationsveranstaltung mit Lungenfacharzt ( 6 x 45 Minuten je 3 Unterstufen mit Lehrern), steht allen Schülern in den Pausen Informationsmaterial des Lungentages zur Verfügung.

4. Parallel - Schnupperkurs „Lungensport“ mit SportlehrerIn „Gleiche Chancen für Schüler mit Asthma“22.

5. Stundenplanänderungen werden von der Schulleitung angeordnet.

Dezember 2007 erhielt ich das vom Vorsitzenden des Prüfungsausschusses der Hochschule Magdeburg, Prof. Dr. Göpel, unterzeichnete Zertifikat über „Kommunales Gesundheitsmanagement".

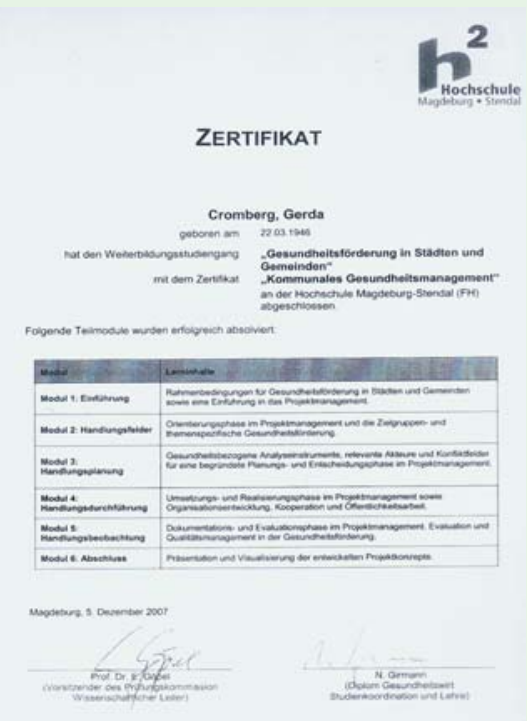

\section{Evaluation/Qualitätsmanagement}

Das Projekt wird von Schülern und Betrieben interessiert angenommen. Das Berufskolleg www.bkherne.de ist Europaschule, Träger des „Schulentwicklungspreises NRW 2008 - „Gute gesunde Schule 2008/2009“ und des Berufswahlsiegels NRW 2009²3.

Gerda Cromberg, Herne

202007 mit Beteiligung der Patientenliga und einem Informationsstand in den Pausen.

${ }^{21}$ Lungentag 2008 - ohne Beteiligung der Patientenliga - begleitet von Dr. Darwiche, seinerzeit EVK Herne.

22 www.atemwegsliga.de (Aktuelles) - Gleiche Chancen - Asthma in der Schule; Frau Tornero Reha-Klinik Hagen-Ambrock übte mit einer Gruppe 2007 in der Sporthalle der Schule „Jazz-Tanz“. 2008 und 2009 war und ist seitens der Sportlehrer kein Schnupperkurs „Lungensport für alle“ geplant.

${ }^{23}$ Meine Aktion am Gesundheitstag Juni 2009 am BK Herne: „Tabakprävention für Jugendliche und junge Erwachsene mit PowerPointpräsentation/ Quiz“ mit Unterlagen des Lungentages und der Broschüre von Prof. Batra „Nichtraucher in 6 Wochen“.

Der Lungentag findet am 30. September 2009 in Herne am BK statt. Begleitung: Dr. med. Darwiche, Ruhrlandklinik Essen. 